\title{
ON LÉVY'S BROWNIAN MOTION INDEXED BY THE ELEMENTS OF COMPACT GROUPS
}

\author{
PAOLO BALDI AND MAURIZIA ROSSI
}

\begin{abstract}
We investigate positive definiteness of the Brownian kernel $K(x, y)=$ $\frac{1}{2}\left(d\left(x, x_{0}\right)+d\left(y, x_{0}\right)-d(x, y)\right)$ on a compact group $G$ and in particular for $G=$ $S O(n)$.
\end{abstract}

\section{INTRODUCTION}

In 1959 P.Lévy [6] asked the question of the existence of a process $X$ indexed by the points of a metric space $(\mathscr{X}, d)$ and generalizing the Brownian motion, i.e. of a real Gaussian process which would be centered, vanishing at some point $x_{0} \in \mathscr{X}$ and such that $\mathbb{E}\left(\left|X_{x}-X_{y}\right|^{2}\right)=d(x, y)$. By polarization, the covariance function of such a process would be

$$
K(x, y)=\frac{1}{2}\left(d\left(x, x_{0}\right)+d\left(y, x_{0}\right)-d(x, y)\right)
$$

so that this question is equivalent to the fact that the kernel $K$ is positive definite. Positive definiteness of $K$ for $\mathscr{X}=\mathbb{R}^{m}$ and $d$ the Euclidean metric had been proved by Schoenberg 14 in 1938 and P.Lévy itself constructed the Brownian motion on $\mathscr{X}=\mathbb{S}^{m-1}$, the euclidean sphere of $\mathbb{R}^{m}, d$ being the distance along the geodesics. Later Gangolli [12] gave an analytical proof of the positive definiteness of the kernel (1.1) for the same metric space $\left(\mathbb{S}^{m-1}, d\right)$, in a paper that dealt with this question for a large class of homogeneous spaces.

Finally Takenaka in [13] proved the positive definiteness of the kernel (1.1) for the Riemannian metric spaces of constant sectional curvature equal to $-1,0$ or 1 , therefore adding the hyperbolic disk to the list. To be precise in the case of the hyperbolic space $\mathcal{H}_{m}=\left\{\left(x_{0}, x_{1}, \ldots, x_{m}\right) \in \mathbb{R}^{m+1}: x_{1}^{2}+\ldots x_{m}^{2}-x_{0}^{2}=1\right\}$, the distance under consideration is the unique, up to multiplicative constants, Riemannian distance that is invariant with respect to the action of $G=L_{m}$, the Lorentz group.

In this short note we investigate this question for the cases $\mathscr{X}=S O(n)$. The answer is that the kernel (1.1) is not positive definite on $S O(n)$ for $n>2$. This is somehow surprising as, in particular, $S O(3)$ is locally isometric to $S U(2)$, where positive definiteness of the kernel $K$ is immediate as shown below.

2010 Mathematics Subject Classification. Primary 43A35; Secondary 60G60, 60B15.

Key words and phrases. positive definite functions, Brownian motion, compact groups. Research supported by ERC grant 277742 Pascal. 
We have been led to the question of the existence of the Brownian motion indexed by the elements of these groups - in particular of $S O(3)$ - in connection with the analysis and the modeling of the Cosmic Microwave Background which has become recently an active research field (see [5], [7], 8], [9] e.g.) and that has attracted the attention to the study of random fields ([1], [2], [11] e.g.). More precisely, in the modern cosmological models the $\mathrm{CMB}$ is seen as the realization of an invariant random field in a vector bundle over the sphere $\mathbb{S}^{2}$ and the analysis of its components (the polarization e.g.) requires the spin random fields theory. This leads naturally to the investigation of invariant random fields on $S O(3)$ enjoying particular properties and therefore to the question of the existence of a privileged random field i.e. Lévy's Brownian random field on $S O(3)$.

In $₫ 2$ we recall some elementary facts about invariant distances and positive definite kernels. In $\$ 3$ we treat the case $G=S U(2)$, recalling well known facts about the invariant distance and Haar measure of this group. Positive definiteness of $K$ for $S U(2)$ is just a simple remark, but these facts are needed in $\$ 4$ where we treat the case $S O(3)$ and deduce from the case $S O(n), n \geq 3$.

\section{Some ELEMENTARY FACTS}

In this section we recall some well known facts about Lie groups (see mainly [3] and also [4], [15]).

2.1. Invariant distance of a compact Lie group. From now on we denote by $G$ a compact Lie group. It is well known that $G$ admits at least a bi-invariant Riemannian metric (see [4] p.66 e.g.), that we shall denote $\left\{\langle\cdot, \cdot\rangle_{g}\right\}_{g \in G}$ where of course $\langle\cdot, \cdot\rangle_{g}$ is the inner product defined on the tangent space $T_{g} G$ to the manifold $G$ at $g$ and the family $\left\{\langle\cdot, \cdot\rangle_{g}\right\}_{g \in G}$ smoothly depends on $g$. By the bi-invariance property, for $g \in G$ the diffeomorphisms $L_{g}$ and $R_{g}$ (resp. the left multiplication and the right multiplication of the group) are isometries. Since the tangent space $T_{g} G$ at any point $g$ can be translated to the tangent space $T_{e} G$ at the identity element $e$ of the group, the metric $\left\{\langle\cdot, \cdot\rangle_{g}\right\}_{g \in G}$ is completely characterized by $\langle\cdot, \cdot\rangle_{e}$. Moreover, $T_{e} G$ being the Lie algebra $\mathfrak{g}$ of $G$, the bi-invariant metric corresponds to an inner product $\langle\cdot, \cdot\rangle$ on $\mathfrak{g}$ which is invariant under the adjoint representation $A d$ of $G$. Indeed there is a one-toone correspondence between bi-invariant Riemannian metrics on $G$ and $A d$-invariant inner products on $\mathfrak{g}$. If in addition $\mathfrak{g}$ is semisimple, then the negative Killing form of $G$ is an $A d$-invariant inner product on $\mathfrak{g}$ itself.

If there exists a unique (up to a multiplicative factor) bi-invariant metric on $G$ (for a sufficient condition see [4], Th. 2.43) and $\mathfrak{g}$ is semisimple, then this metric is necessarily proportional to the negative Killing form of $\mathfrak{g}$. It is well known that this is the case for $S O(n),(n \neq 4)$ and $S U(n)$; furthermore, the (natural) Riemannian metric on $S O(n)$ induced by the embedding $S O(n) \hookrightarrow \mathbb{R}^{n^{2}}$ corresponds to the negative Killing form of so(n).

Endowed with this bi-invariant Riemannian metric, $G$ becomes a metric space, with a distance $d$ which is bi-invariant. Therefore the function $g \in G \rightarrow d(g, e)$ is a class 
function as

$$
d(g, e)=d(h g, h)=d\left(h g h^{-1}, h h^{-1}\right)=d\left(h g h^{-1}, e\right), \quad g, h \in G .
$$

It is well known that geodesics on $G$ through the identity $e$ are exactly the one parameter subgroups of $G$ (see [10] p.113 e.g.), thus a geodesic from $e$ is the curve on $G$

$$
\gamma_{X}(t): t \in[0,1] \rightarrow \exp (t X)
$$

for some $X \in \mathfrak{g}$. The length of this geodesic is

$$
L\left(\gamma_{X}\right)=\|X\|=\sqrt{\langle X, X\rangle} .
$$

Therefore

$$
d(g, e)=\inf _{X \in \mathfrak{g}: \exp X=g}\|X\| .
$$

2.2. Brownian kernels on a metric space. Let $(\mathscr{X}, d)$ be a metric space.

Lemma 2.1. The kernel $K$ in (1.1) is positive definite on $\mathscr{X}$ if and only if $d$ is a restricted negative definite kernel, i.e., for every choice of elements $x_{1}, \ldots, x_{n} \in \mathscr{X}$ and of complex numbers $\xi_{1}, \ldots, \xi_{n}$ with $\sum_{i=1}^{n} \xi_{i}=0$

$$
\sum_{i, j=1}^{n} d\left(x_{i}, x_{j}\right) \xi_{i} \overline{\xi_{j}} \leq 0
$$

Proof. For every $x_{1}, \ldots, x_{n} \in \mathscr{X}$ and complex numbers $\xi_{1}, \ldots, \xi_{n}$

$$
\sum_{i, j} K\left(x_{i}, x_{j}\right) \xi_{i} \overline{\xi_{j}}=\frac{1}{2}\left(\bar{a} \sum_{i} d\left(x_{i}, x_{0}\right) \xi_{i}+a \sum_{j} d\left(x_{j}, x_{0}\right) \overline{\xi_{j}}-\sum_{i, j} d\left(x_{i}, x_{j}\right) \xi_{i} \overline{\xi_{j}}\right)
$$

where $a:=\sum_{i} \xi_{i}$. If $a=0$ then it is immediate that in (2.3) the 1.h.s. is $\geq 0$ if and only if the r.h.s. is $\leq 0$. Otherwise set $\xi_{0}:=-a$ so that $\sum_{i=0}^{n} \xi_{i}=0$. The following equality

$$
\sum_{i, j=0}^{n} K\left(x_{i}, x_{j}\right) \xi_{i} \overline{\xi_{j}}=\sum_{i, j=1}^{n} K\left(x_{i}, x_{j}\right) \xi_{i} \overline{\xi_{j}}
$$

is then easy to check, keeping in mind that $K\left(x_{i}, x_{0}\right)=K\left(x_{0}, x_{j}\right)=0$, which finishes the proof.

For a more general proof see [12] p. 127 in the proof of Lemma 2.5.

If $\mathscr{X}$ is the homogeneous space of some topological group $G$, and $d$ is a $G$-invariant distance, then (2.2) is satisfied if and only if for every choice of elements $g_{1}, \ldots, g_{n} \in G$ and of complex numbers $\xi_{1}, \ldots, \xi_{n}$ with $\sum_{i=1}^{n} \xi_{i}=0$

$$
\sum_{i, j=1}^{n} d\left(g_{i} g_{j}^{-1} x_{0}, x_{0}\right) \xi_{i} \overline{\xi_{j}} \leq 0
$$

where $x_{0} \in \mathscr{X}$ is a fixed point. We shall say that the function $g \in G \rightarrow d\left(g x_{0}, x_{0}\right)$ is restricted negative definite on $G$ if it satisfies (2.5). 
In our case of interest $\mathscr{X}=G$ a compact (Lie) group and $d$ is a bi-invariant distance as in $§ 2.1$. The Peter-Weyl development (see [3] e.g.) for the class function $d(\cdot, e)$ on $G$ is

$$
d(g, e)=\sum_{\ell \in \widehat{G}} \alpha_{\ell} \chi_{\ell}(g)
$$

where $\widehat{G}$ denotes the family of equivalence classes of irreducible representations of $G$ and $\chi_{\ell}$ the character of the $\ell$-th irreducible representation of $G$.

Remark 2.2. A function $\phi$ with a development as in (2.6) is restricted negative definite if and only if $\alpha_{\ell} \leq 0$ but for the trivial representation.

Actually note first that, by standard approximation arguments, $\phi$ is restricted negative definite if and only if for every continuous function $f: G \rightarrow \mathbb{C}$ with 0 -mean (i.e. orthogonal to the constants)

$$
\int_{G} \int_{G} \phi\left(g h^{-1}\right) f(g) \overline{f(h)} d g d h \leq 0
$$

$d g$ denoting the Haar measure of $G$. Choosing $f=\chi_{\ell}$ in the l.h.s. of (2.7) and denoting $d_{\ell}$ the dimension of the corresponding representation, a straightforward computation gives

$$
\int_{G} \int_{G} \phi\left(g h^{-1}\right) \chi_{\ell}(g) \overline{\chi_{\ell}(h)} d g d h=\frac{\alpha_{\ell}}{d_{\ell}}
$$

so that if $\phi$ restricted negative definite, $\alpha_{\ell} \leq 0$ necessarily.

Conversely, if $\alpha_{\ell} \leq 0$ but for the trivial representation, then $\phi$ is restricted negative definite, as the characters $\chi_{\ell}$ 's are positive definite and orthogonal to the constants.

\section{3. $S U(2)$}

The special unitary group $S U(2)$ consists of the complex unitary $2 \times 2$-matrices $g$ such that $\operatorname{det}(g)=1$. Every $g \in S U(2)$ has the form

$$
g=\left(\begin{array}{cc}
a & b \\
-\bar{b} & \bar{a}
\end{array}\right), \quad a, b \in \mathbb{C},|a|^{2}+|b|^{2}=1 .
$$

If $a=a_{1}+i a_{2}$ and $b=b_{1}+i b_{2}$, then the map

$$
\Phi(g)=\left(a_{1}, a_{2}, b_{1}, b_{2}\right)
$$

is an homeomorphism (see [3], [15] e.g.) between $S U(2)$ and the unit sphere $\mathbb{S}^{3}$ of $\mathbb{R}^{4}$. Moreover the right translation

$$
R_{g}: h \rightarrow h g, \quad h, g \in S U(2)
$$

of $S U(2)$ is a rotation (an element of $S O(4)$ ) of $\mathbb{S}^{3}$ (identified with $S U(2)$ ). The homeomorphism (3.2) preserves the invariant measure, i.e., if $d g$ is the normalized 
Haar measure on $S U(2)$, then $\Phi(d g)$ is the normalized Lebesgue measure on $\mathbb{S}^{3}$. As the 3-dimensional polar coordinates on $\mathbb{S}^{3}$ are

$$
\begin{aligned}
& a_{1}=\cos \theta, \\
& a_{2}=\sin \theta \cos \varphi, \\
& b_{1}=\sin \theta \sin \varphi \cos \psi, \\
& b_{2}=\sin \theta \sin \varphi \sin \psi,
\end{aligned}
$$

$(\theta, \varphi, \psi) \in[0, \pi] \times[0, \pi] \times[0,2 \pi]$, the normalized Haar integral of $S U(2)$ for an integrable function $f$ is

$$
\int_{S U(2)} f(g) d g=\frac{1}{2 \pi^{2}} \int_{0}^{\pi} \sin \varphi d \varphi \int_{0}^{\pi} \sin ^{2} \theta d \theta \int_{0}^{2 \pi} f(\theta, \varphi, \psi) d \psi
$$

The bi-invariant Riemannian metric on $S U(2)$ is necessarily proportional to the negative Killing form of its Lie algebra su(2) (the real vector space of $2 \times 2$ anti-hermitian complex matrices). We consider the bi-invariant metric corresponding to the $A d$ invariant inner product on $s u(2)$

$$
\langle X, Y\rangle=-\frac{1}{2} \operatorname{tr}(X Y), \quad X, Y \in s u(2) .
$$

Therefore as an orthonormal basis of $s u(2)$ we can consider the matrices

$$
X_{1}=\left(\begin{array}{cc}
0 & 1 \\
-1 & 0
\end{array}\right), \quad X_{2}=\left(\begin{array}{cc}
0 & i \\
i & 0
\end{array}\right), \quad X_{3}=\left(\begin{array}{cc}
i & 0 \\
0 & -i
\end{array}\right)
$$

The homeomorphism (3.2) is actually an isometry between $S U(2)$ endowed with this distance and $\mathbb{S}^{3}$. Hence the restricted negative definiteness of the kernel $d$ on $S U(2)$ is an immediate consequence of this property on $\mathbb{S}^{3}$ which is known to be true as mentioned in the introduction ([12], [6], [13]). In order to develop a comparison with $S O(3)$, we shall give a different proof of this fact in $\$ 5$.

$$
\text { 4. } S O(n)
$$

We first investigate the case $n=3$. The group $S O(3)$ can also be realized as a quotient of $S U(2)$. Actually the adjoint representation $A d$ of $S U(2)$ is a surjective morphism from $S U(2)$ onto $S O(3)$ with kernel $\{ \pm e\}$ (see [3] e.g.). Hence the well known result

$$
S O(3) \cong S U(2) /\{ \pm e\} .
$$

Let us explicitly recall this morphism: if $a=a_{1}+i a_{2}, b=b_{1}+i b_{2}$ with $|a|^{2}+|b|^{2}=1$ and

$$
\widetilde{g}=\left(\begin{array}{cc}
a & b \\
-\bar{b} & \bar{a}
\end{array}\right)
$$

then the orthogonal matrix $A d(\widetilde{g})$ is given by

$$
g=\left(\begin{array}{ccc}
a_{1}^{2}-a_{2}^{2}-\left(b_{1}^{2}-b_{2}^{2}\right) & -2 a_{1} a_{2}-2 b_{1} b_{2} & -2\left(a_{1} b_{1}-a_{2} b_{2}\right) \\
2 a_{1} a_{2}-2 b_{1} b_{2} & \left(a_{1}^{2}-a_{2}^{2}\right)+\left(b_{1}^{2}-b_{2}^{2}\right) & -2\left(a_{1} b_{2}+a_{2} b_{1}\right) \\
2\left(a_{1} b_{1}+a_{2} b_{2}\right) & -2\left(-a_{1} b_{2}+a_{2} b_{1}\right) & |a|^{2}-|b|^{2}
\end{array}\right)
$$


The isomorphism in (4.1) might suggest that the positive definiteness of the Brownian kernel on $S U(2)$ implies a similar result for $S O(3)$. This is not true and actually it turns out that the distance $(g, h) \rightarrow d(g, h)$ on $S O(3)$ induced by its bi-invariant Riemannian metric is not a restricted negative definite kernel (see Lemma 2.1).

As for $S U(2)$, the bi-invariant Riemannian metric on $S O(3)$ is proportional to the negative Killing form of its Lie algebra so(3) (the real $3 \times 3$ antisymmetric real matrices). We shall consider the $A d$-invariant inner product on $s o(3)$ defined as

$$
\langle A, B\rangle=-\frac{1}{2} \operatorname{tr}(A B), \quad A, B \in s o(3) .
$$

An orthonormal basis for $s o(3)$ is therefore given by the matrices

$$
A_{1}=\left(\begin{array}{ccc}
0 & 0 & 0 \\
0 & 0 & -1 \\
0 & 1 & 0
\end{array}\right), \quad A_{2}=\left(\begin{array}{ccc}
0 & 0 & 1 \\
0 & 0 & 0 \\
-1 & 0 & 0
\end{array}\right), \quad A_{3}=\left(\begin{array}{ccc}
0 & -1 & 0 \\
1 & 0 & 0 \\
0 & 0 & 0
\end{array}\right)
$$

Similarly to the case of $S U(2)$, it is easy to compute the distance from $g \in S O(3)$ to the identity. Actually $g$ is conjugated to the matrix of the form

$$
\Delta(t)=\left(\begin{array}{ccc}
\cos t & \sin t & 0 \\
-\sin t & \cos t & 0 \\
0 & 0 & 1
\end{array}\right)=\exp \left(t A_{1}\right)
$$

where $t \in[0, \pi]$ is the rotation angle of $g$. Therefore if $d$ still denotes the distance induced by the bi-invariant metric,

$$
d(g, e)=d(\Delta(t), e)=t
$$

i.e. the distance from $g$ to $e$ is the rotation angle of $g$.

Let us denote $\left\{\chi_{\ell}\right\}_{\ell \geq 0}$ the set of characters for $S O(3)$. It is easy to compute the Peter-Weyl development in (2.6) for $d(\cdot, e)$ as the characters $\chi_{\ell}$ are also simple functions of the rotation angle. More precisely, if $t$ is the rotation angle of $g$ (see [8] e.g.),

$$
\chi_{\ell}(g)=\frac{\sin \frac{(2 \ell+1) t}{2}}{\sin \frac{t}{2}}=1+2 \sum_{m=1}^{\ell} \cos (m t) .
$$

We shall prove that the coefficient

$$
\alpha_{\ell}=\int_{S O(3)} d(g, e) \chi_{\ell}(g) d g
$$

is positive for some $\ell \geq 1$. As both $d(\cdot, e)$ and $\chi_{\ell}$ are functions of the rotation angle $t$, we have

$$
\alpha_{\ell}=\int_{0}^{\pi} t\left(1+2 \sum_{j=1}^{\ell} \cos (j t)\right) p_{T}(t) d t
$$

where $p_{T}$ is the density of $t=t(g)$, considered as a r.v. on the probability space $(S O(3), d g)$. The next statements are devoted to the computation of the density $p_{T}$. 
This is certainly well known but we were unable to find a reference in the literature. We first compute the density of the trace of $g$.

Proposition 4.1. The distribution of the trace of a matrix in $S O(3)$ with respect to the normalized Haar measure is given by the density

$$
f(y)=\frac{1}{2 \pi}(3-y)^{1 / 2}(y+1)^{-1 / 2} 1_{[-1,3]}(y) .
$$

Proof. The trace of the matrix (4.2) is equal to

$$
\operatorname{tr}(g)=3 a_{1}^{2}-a_{2}^{2}-b_{1}^{2}-b_{2}^{2} .
$$

Under the normalized Haar measure of $S U(2)$ the vector $\left(a_{1}, a_{2}, b_{1}, b_{2}\right)$ is uniformly distributed on the sphere $\mathbb{S}^{3}$. Recall the normalized Haar integral (3.4) so that, taking the corresponding marginal, $\theta$ has density

$$
f_{1}(\theta)=\frac{2}{\pi} \sin ^{2}(\theta) d \theta
$$

Now

$$
3 a_{1}^{2}-a_{2}^{2}-b_{1}^{2}-b_{2}^{2}=4 \cos ^{2} \theta-1 .
$$

Let us first compute the density of $Y=\cos ^{2} X$, where $X$ is distributed according to the density (4.4). This is elementary as

$F_{Y}(t)=\mathbb{P}\left(\cos ^{2} X \leq t\right)=\mathbb{P}(\arccos (\sqrt{t}) \leq X \leq \arccos (-\sqrt{t}))=\frac{2}{\pi} \int_{\arccos (\sqrt{t})}^{\arccos (-\sqrt{t})} \sin ^{2}(\theta) d \theta$.

Taking the derivative it is easily found that the density of $Y$ is, for $0<t<1$,

$$
F_{Y}^{\prime}(t)=\frac{2}{\pi}(1-t)^{1 / 2} t^{-1 / 2}
$$

By an elementary change of variable the distribution of the trace $4 Y-1$ is therefore given by (4.3).

Corollary 4.2. The distribution of the rotation angle of a matrix in $S O(3)$ is

$$
p_{T}(t)=\frac{1}{\pi}(1-\cos t) 1_{[0, \pi]}(t) .
$$

Proof. It suffices to remark that if $t$ is the rotation angle of $g$, then its trace is equal to $2 \cos t+1 . p_{T}$ is therefore the distribution of $W=\arccos \left(\frac{Y-1}{2}\right), Y$ being distributed as (4.3). The elementary details are left to the reader.

Now it is easy to compute the Fourier development of the function $d(\cdot, e)$.

Proposition 4.3. The kernel d on $S O(3)$ is not restricted negative definite. 
Proof. It is enough to show that in the Fourier development

$$
d(g, e)=\sum_{\ell \geq 0} \alpha_{\ell} \chi_{\ell}(g)
$$

$\alpha_{\ell}>0$ for some $\ell \geq 1$ (see Remark 2.2). We have

$$
\begin{gathered}
\alpha_{\ell}=\int_{S O(3)} d(g, e) \chi_{\ell}(g) d g=\frac{1}{\pi} \int_{0}^{\pi} t\left(1+2 \sum_{m=1}^{\ell} \cos (m t)\right)(1-\cos t) d t= \\
=\frac{1}{\pi} \underbrace{\int_{0}^{\pi} t(1-\cos t) d t}_{:=I_{1}}+\frac{2}{\pi} \sum_{m=1}^{\ell} \underbrace{\int_{0}^{\pi} t \cos (m t) d t}_{:=I_{2}}-\frac{2}{\pi} \sum_{m=1}^{\ell} \underbrace{\int_{0}^{\pi} t \cos (m t) \cos t d t}_{:=I_{3}} .
\end{gathered}
$$

Now integration by parts gives

$$
I_{1}=\frac{\pi^{2}}{2}+2, \quad I_{2}=\frac{(-1)^{m}-1}{m^{2}},
$$

whereas, if $m \neq 1$, we have

$$
I_{3}=\int_{0}^{\pi} t \cos (m t) \cos t d t=\frac{m^{2}+1}{\left(m^{2}-1\right)^{2}}\left((-1)^{m}+1\right)
$$

and for $m=1$,

$$
I_{3}=\int_{0}^{\pi} t \cos ^{2} t d t=\frac{\pi^{2}}{4} .
$$

Putting things together we find

$$
\alpha_{\ell}=\frac{2}{\pi}\left(1+\sum_{m=1}^{\ell} \frac{(-1)^{m}-1}{m^{2}}+\sum_{m=2}^{\ell} \frac{m^{2}+1}{\left(m^{2}-1\right)^{2}}\left((-1)^{m}+1\right)\right) .
$$

If $\ell=2$, for instance, we find $\alpha_{2}=\frac{2}{9 \pi}>0$, but it is easy to see that $\alpha_{\ell}>0$ for every $\ell$ even.

Consider now the case $n>3$. $S O(n)$ contains a closed subgroup $H$ that is isomorphic to $S O(3)$ and the restriction to $H$ of any bi-invariant distance $d$ on $S O(n)$ is a bi-invariant distance $\widetilde{d}$ on $S O(3)$. By Proposition 4.3, $\widetilde{d}$ is not restricted negative definite, therefore there exist $g_{1}, g_{2}, \ldots, g_{m} \in H, \xi_{1}, \xi_{2}, \ldots, \xi_{m} \in \mathbb{R}$ with $\sum_{i=1}^{m} \xi_{i}=0$ such that

$$
\sum_{i, j} d\left(g_{i}, g_{j}\right) \xi_{i} \xi_{j}=\sum_{i, j} \widetilde{d}\left(g_{i}, g_{j}\right) \xi_{i} \xi_{j}>0
$$

We have therefore

Corollary 4.4. Any bi-invariant distance $d$ on $S O(n), n \geq 3$ is not a restricted negative definite kernel. 
Remark that the bi-invariant Riemannian metric on $S O(4)$ is not unique, meaning that it is not necessarily proportional to the negative Killing form of $s o(4)$. In this case Corollary 4.4 states that every such bi-invariant distance cannot be restricted negative definite.

\section{FINAL REMARKS}

We were intrigued by the different behavior of the invariant distance of $S U(2)$ and $S O(3)$ despite these groups are locally isometric and decided to compute also for $S U(2)$ the development

$$
d(g, e)=\sum_{\ell} \alpha_{\ell} \chi_{\ell}(g)
$$

This is not difficult as, denoting by $t$ the distance of $g$ from $e$, the characters of $S U(2)$ are

$$
\chi_{\ell}(g)=\frac{\sin ((\ell+1) t)}{\sin t}, \quad t \neq k \pi
$$

and $\chi_{\ell}(e)=\ell+1$ if $t=0, \chi_{\ell}(-)=(-1)^{\ell}(\ell+1)$ if $t=\pi$. Then it is elementary to compute, for $\ell>0$,

$$
\alpha_{\ell}=\frac{1}{\pi} \int_{0}^{\pi} t \sin ((\ell+1) t) \sin t d t= \begin{cases}-\frac{8}{\pi} \frac{m+1}{m^{2}(m+2)^{2}} & \ell \text { odd } \\ 0 & \ell \text { even }\end{cases}
$$

thus confirming the restricted negative definiteness of $d$ (see Remark 2.2). Remark also that the coefficients corresponding to the even numbered representations, that are also representations of $S O(3)$, here vanish.

Acknowledgements. The authors wish to thank A.Iannuzzi and S.Trapani for valuable assistance.

\section{REFERENCES}

[1] P. Baldi and D. Marinucci, Some characterizations of the spherical harmonics coefficients for isotropic random fields, Statist. Probab. Lett. 77 (2007), no. 5, 490-496.

[2] P. Baldi, D. Marinucci, and V. S. Varadarajan, On the characterization of isotropic Gaussian fields on homogeneous spaces of compact groups, Electron. Comm. Probab. 12 (2007), 291-302 (electronic).

[3] J. Faraut, Analysis on Lie groups, Cambridge Studies in Advanced Mathematics, vol. 110, Cambridge University Press, Cambridge, 2008.

[4] S. Gallot, D. Hulin, and J. Lafontaine, Riemannian geometry, third ed., Universitext, SpringerVerlag, Berlin, 2004.

[5] N. Leonenko and L. Sakhno, On spectral representations of tensor random fields on the sphere, Stoch. Anal. Appl. 30 (2012), no. 1, 44-66.

[6] P. Lévy, Le mouvement brownien fonction d'un point de la sphére de Riemann, Rend. Circ. Mat. Palermo 8 (1959), 297-310.

[7] A. Malyarenko, Invariant random fields in vector bundles and application to cosmology, Ann. Inst. Henri Poincaré Probab. Stat. 47 (2011), no. 4, 1068-1095. 
[8] D. Marinucci and G. Peccati, Random Fields, London Mathematical Society Lecture Note Series 389, Cambridge University Press, Cambridge, 2011.

[9] D. Marinucci and I. Wigman, On the area of excursion sets of spherical Gaussian eigenfunctions, J. Math. Phys. 52 (2011), no. 9, 093301, 21.

[10] J. Milnor, Morse theory, Based on lecture notes by M. Spivak and R. Wells. Annals of Mathematics Studies, No. 51, Princeton University Press, Princeton, N.J., 1963.

[11] G. Peccati and J.-R. Pycke, Decompositions of stochastic processes based on irreducible group representations, Teor. Veroyatn. Primen. 54 (2009), no. 2, 304-336.

[12] R.Gangolli, Positive definite kernels on homogeneous spaces and certain sthocastic processes related to lévy's brownian motion of several parameters, Ann. I l'I.H.P. section B, tome 3, $\mathbf{n}$. 2 (1967), 121-226.

[13] I. Kubo S. Takenaka and H. Urakawa, Brownian motion parametrized with metric space of constant curvature, Nagoya Math. J. 82 (1981), 131-140.

[14] I. J. Schoenberg, Metric spaces and positive definite functions, Trans. Amer. Math. Soc. 44 (1938), no. 3, 522-536.

[15] M. Sugiura, Unitary representations and harmonic analysis, second ed., North-Holland Mathematical Library, vol. 44, North-Holland Publishing Co., Amsterdam, 1990.

Dipartimento di Matematica, Università di Roma Tor Vergata, 00133 Roma, Italy

E-mail address: baldi@mat.uniroma2.it

Dipartimento di MATEmaticA, Università Di Roma Tor Vergata, 00133 Roma, Italy

E-mail address: rossim@mat.uniroma2.it 